\title{
Quantitative depth profiling of deuterium up to very large depths
}

\author{
M. Mayer ${ }^{\mathrm{a}}$, E. Gauthier ${ }^{\mathrm{b}}$, K. Sugiyama ${ }^{\mathrm{a}}$, and U. von Toussaint ${ }^{\mathrm{a}}$ \\ ${ }^{a}$ Max-Planck-Institut für Plasmaphysik, EURATOM Association, Boltzmannstr. 2, \\ D-85748 Garching, Germany \\ ${ }^{\mathrm{b}}$ Association EURATOM-CEA Cadarache, 13018 St. Paul lez Durance, France
}

\begin{abstract}
Quantitative depth profiles of deuterium up to very large depths are achieved from the energy spectra of protons created by the $\mathrm{D}\left({ }^{3} \mathrm{He}, \mathrm{p}\right) \alpha$ nuclear reaction at incident energies up to $6 \mathrm{MeV}$. The advantages of this method compared to the more often applied resonance method are discussed. For light target materials the achievable depth resolution is mainly limited by geometrical spread due to the finite size of the detector aperture, while for heavy materials the resolution is mainly limited by multiple small-angle scattering. A reasonable depth resolution throughout the whole analyzed depth can be obtained by using several different incident energies. Depth profiling up to $38 \mu \mathrm{m}$ is demonstrated for a-C:D layers deposited on the limiter of Tore Supra, and up to $7.5 \mu \mathrm{m}$ in tungsten coatings from the divertor of ASDEX Upgrade.
\end{abstract}

Key words: Depth profiling, Deuterium, Nuclear reaction analysis PACS: 81.70.Jb, 82.80.Yc, 29.30.Ep, 25.55.-e, 29.85.-c

\section{Introduction}

The use of carbon fiber composites (CFC), molybdenum and tungsten as plasma-facing materials in nuclear fusion research resulted in an increasing interest in quantitative depth profiling of deuterium up to very large depths. Deuterium can penetrate in these materials up to depths of several tens to several hundreds of $\mu \mathrm{m}$, where it is trapped at low concentrations [1,2]. It has been assumed that this deep penetration is an important process for the deuterium inventory in the tokamak Tore Supra [3], and it has been shown that deep penetration is dominating the remaining deuterium inventory in the all-tungsten tokamak ASDEX Upgrade [4]. In carbon materials this penetration is related to the porosity of the material, while in tungsten the diffusion 
process is driven by the stress-field induced by implanted deuterium [2]. These processes are only poorly understood, and their investigation requires methods for quantitative depth profiling of deuterium up to depths of at least ten (or better a few ten) $\mu \mathrm{m}$. The investigated samples often originate from wall elements of fusion experiments and have technical, rough surfaces. Consequently the applied depth profiling methods should be as little sensitive to surface roughness as possible, prohibiting grazing incident or exit angles for ion beam analysis methods.

Secondary ion mass spectroscopy (SIMS) allows depth profiling of hydrogen isotopes up to a depth of about $100 \mu \mathrm{m}$ [5], but it is time consuming and not quantitative by itself. A quantification can be obtained by comparison with ion beam analysis results for the surface layer. For good accuracy the ion-beam analyzed layer should be as thick as possible, which again raises the question of quantitative hydrogen isotopes analysis up to larger depths.

Neutron-ERDA (NERDA) with incident $14 \mathrm{MeV}$ neutrons from the sample back side allows depth profiling of hydrogen isotopes up to depths of hundreds of $\mu \mathrm{m}$ [6-8]. However, the depth resolution of this method is only a few ten to about $100 \mu \mathrm{m}[6,8]$ and counting statistics is poor due to the low incident neutron fluxes, resulting in low sensitivity [6].

The $\mathrm{D}(\mathrm{d}, \mathrm{p}) \mathrm{T}$ and the $\mathrm{D}\left({ }^{3} \mathrm{He}, \mathrm{p}\right) \alpha$ reactions can be used for quantitative depth profiling of $\mathrm{D}$ up to large depths. The $\mathrm{D}(\mathrm{d}, \mathrm{p}) \mathrm{T}$ reaction has a $\mathrm{Q}$-value of 4.03 MeV, resulting in proton energies in the range of 2.1-2.4 MeV at backward angles. This allows the detection of deuterium up to a maximum depth of about $20 \mu \mathrm{m}$ in carbon and about $6 \mu \mathrm{m}$ in tungsten: Larger depths are not accessible because the created protons overlap with backscattered deuterons at incident energies above about $2.5 \mathrm{MeV}$, while for lower energies the analyzed depth is limited by the penetration depth of the deuterons. This reaction is also not well suited for targets containing heavy elements due to the high yield of backscattered primary particles.

The $\mathrm{D}\left({ }^{3} \mathrm{He}, \mathrm{p}\right) \alpha$ reaction has been successfully applied for depth profiling of $\mathrm{D}$ during the last three decades [9]. It has a Q-value of $18.4 \mathrm{MeV}$, resulting in proton energies in the range 11-14 MeV. This high proton energy allows easy filtering of backscattered primary particles by a stopper foil. The reaction has inverse kinematics, i.e. the energy of the protons increases if the energy of the incident ${ }^{3} \mathrm{He}$ decreases. Several groups have used the resonance method, which exploits the broad resonance of this reaction by measuring the total proton yield as function of incident energy [10-15]. The resonance method uses only the total number of detected protons and can be utilized with detectors having large solid angles (where the geometrical spread can be huge), and it can be used with thin detectors, where the $14 \mathrm{MeV}$ protons are not fully stopped. In case of overlapping peaks adjustable sensitive zone detectors can 
be used for unscrambling the different peak contributions [16]. However, due to the broad resonance with a FWHM of $730 \mathrm{keV}$ and the non-negligible contribution of protons originating from outside the resonance region this method requires measurements at 8-10 different energies, thus being timeconsuming; it requires good counting statistics, which largely compensates the advantage of a large detector; and it is often difficult to show that the derived depth profile is unambiguous.

Additional information can be obtained from the energy spectra of the emitted protons. This requires thick detectors (depletion depth about $2 \mathrm{~mm}$ ), which are now readily available. Due to the inverse kinematics of the reaction, the large influence of multiple scattering on the incident path, and a large geometrical straggling the depth resolution has several non-standard properties, which are not encountered in common ion beam analysis techniques for depth profiling, such as RBS or ERDA.

Usually the results obtained with one single incident energy do not provide all necessary information, and measurements at different incident energies have to be combined. This requires a more thorough design of measurements than with standard techniques. This paper investigates the depth resolution as function of incident energy and depth for light and heavy materials and presents examples for depth profiling of deuterium in carbon and tungsten.

\section{Experimental}

All measurements were performed at the $3 \mathrm{MV}$ tandem accelerator at the IPP Garching. The proton detector has an area of $300 \mathrm{~mm}^{2}$ and a depletion depth of $2 \mathrm{~mm}$ with a resolution of $22 \mathrm{keV}$, type ORTEC BA 022-300-2000. The detector is located at a reaction angle of $135^{\circ}$ having a distance of about $40 \mathrm{~mm}$ to the target, a schematic representation of the setup is shown in Fig. 1. The initial energy of reaction products is constant on a cone with fixed reaction angle. This constant energy cone intercepts the detector slit plane in a conic section, which is a parabola at the reaction angle of $135^{\circ}$. The kinematic energy spread is minimized if the detector slit follows this conic section [17]. We use a detector aperture with parabolic shape, $3 \mathrm{~mm}$ width and $17 \mathrm{~mm}$ height. For easier manufacture the parabola was approximated as the arc of a circle [17], which introduces a maximum deviation of about $0.05 \mathrm{~mm}$ from the parabola. The stopper foil consists of $5 \mu \mathrm{m}$ Ni combined with $12 \mu \mathrm{m}$ Mylar, which allows to filter ${ }^{3} \mathrm{He}$ ions up to energies of about $5 \mathrm{MeV}$. The incident beam current was about $30 \mathrm{nA}$ on a beam spot of $1 \times 1 \mathrm{~mm}^{2}$. 


\section{Depth resolution calculation and computer simulation}

The depth resolution, as used throughout this paper, is obtained from the effective stopping power $S_{\text {eff, }}$ which is defined by

$$
S_{\mathrm{eff}}(x)=\left|\frac{d E}{d x}(x)\right| .
$$

$E$ is the energy of the detected particles and $x$ the depth of origin, i.e. the depth where the reaction occurred. $S_{\text {eff }}$ merges the stopping powers of incident and outgoing particles and the change of the proton energy with incident energy. The depth resolution $\Delta x$ is then obtained from

$$
\Delta x(x)=\frac{\Delta E(x)}{S_{\mathrm{eff}}(x)},
$$

with $\Delta E(x)$ the energy spread of detected particles originating from the depth $x . \Delta E$ is the energy spread in full width at half maximum (FWHM), and correspondingly $\Delta x$ is the depth resolution in FWHM. See [18, Section 2] for a discussion of this definition.

Depth resolution calculations were performed with the program RESOLNRA [19] and SIMNRA 6.29 [20]. Energy-loss straggling in the target and stopper foils, energy spread due to multiple scattering in target and foil, geometrical spread due to finite beam size and detector aperture, and detector resolution were taken into account [21,18]. SRIM-2003 stopping powers [22] and crosssection data from [15] were used.

\section{Deuterium in carbon}

As will be shown below, the depth resolution for deuterium in carbon is determined by the geometrical spread due to detector aperture and beam spot size. Consequently, the size of the detector aperture has to be selected with care. The depth resolution for deuterium in carbon is shown in Fig. 2 for an incident energy of $2500 \mathrm{keV}$ and different aperture shapes. The maximum analyzable depth at this energy is about $9 \mu \mathrm{m}^{1}$. The worst depth resolution is always obtained at the surface, while the depth resolution improves in larger depths. This is an unusual property of this reaction, which is normally not observed in other ion beam analysis techniques.

$\overline{1}$ For simplicity we use a carbon density of $10^{23}$ atoms $/ \mathrm{cm}^{3}$. 
Geometrical spread is caused by the finite sizes of detector aperture and beam spot and results in a spread of exit and reaction angles [18, Section 4.2]. The spread of exit angles causes path length differences for emerging particles, thus resulting in energy spread, while the spread of reaction angles results in kinematic energy spread. Spread due to path length differences and kinematic spread are correlated and have to be considered simultaneously. Due to the negligible energy loss of the outgoing protons the energy spread (and hence the depth resolution) is determined by the kinematic spread, which decreases with decreasing incident energy. This results in an improved depth resolution at larger depths. The largest aperture with $17 \mathrm{~mm}$ diameter results in a large solid angle of $142 \mathrm{msr}$, but the depth resolution at the surface is then only about $11 \mu \mathrm{m}$ FWHM: This is not suitable for depth profiling. The parabolic slit (see section 2) improves the depth resolution, but decreases the solid angle and hence the sensitivity. An indefinitely narrow slit and indefinitely small beam spot would eliminate geometrical straggling, resulting in a depth resolution of about $1 \mu \mathrm{m}$ at the target surface. But this would reduce the sensitivity to zero. In our measurements the sensitivity for small deuterium concentrations is more important than depth resolution, so we decided to use a $3 \mathrm{~mm}$ wide slit. This results in a depth resolution of $2.5 \mu \mathrm{m}$ at the surface (at $2500 \mathrm{keV}$ ) and a solid angle of $30.7 \mathrm{msr}$.

The different energy-spread contributions to the depth resolution are shown in Fig. 3. Geometrical straggling is the dominant energy-spread mechanism close to the target surface, while multiple small-angle scattering becomes dominant at depths $>5 \mu \mathrm{m}$. Energy-loss straggling (mainly on the incident path) and energy-loss straggling in the stopper foil add some energy spread, while the detector energy resolution is negligible.

The uncommon property of a low depth resolution close to the surface, but a better depth resolution deeper in the sample, offers the possibility to obtain an acceptable depth resolution throughout the whole analyzed depth by using different incident energies. This is shown in Fig. 4 for incident energies from $500-6000 \mathrm{keV}$. With energies in the range 500-1000 keV the top $2 \mu \mathrm{m}$ of the sample can be probed with a resolution of about $0.2 \mu \mathrm{m}$. Larger depths require higher incident energies. These yield an unfeasible depth resolution close to the surface, but the depth resolution improves at larger depths. A depth resolution better than $1 \mu \mathrm{m}$ can be obtained up to a maximum analyzable depth of about $32 \mu \mathrm{m}$. A continuous depth resolution better than $1 \mu \mathrm{m}$ throughout the whole analyzable depth requires measurements at about 8 different incident energies. A better depth resolution can be obtained with a narrower detector aperture, but at the cost of a smaller solid angle and smaller sensitivity, see Fig. 2. Coincidentally the best depth resolution is obtained close to the depth where the cross-section has its maximum (marked by arrows in Fig. 4), i.e. where the highest sensitivity is achieved. The depth resolution of the resonance method, as used in [10-15], is determined by the FWHM of the cross-section maximum, 
which is about $730 \mathrm{keV}$. Assuming a Gaussian shape of the cross-section maximum this gives a depth resolution of about $2 \mu \mathrm{m}$ FWHM throughout the whole analyzed depth, see Fig. 4 . In reality the shape of the cross-section is non-Gaussian with a long tail towards higher energies, so that this resolution is an optimistic limit which will not be fully reached in practical applications. Moreover, this resolution is only obtained if measurements at energies with a step width of the half FWHM of the cross-section maximum, i.e. a step width of $360 \mathrm{keV}$ or less, are performed. This requires measurements at about 15 different incident energies: The resonance method therefore requires twice the number of measurements to achieve a resolution which is inferior by a factor of two, as compared to the depth resolution obtained from the energy spectrum of the protons. The depth resolution of the resonance method can be increased by tilting the sample, but this decreases the maximum analyzable depth. The depth resolution of the proton energy spectrum method can be increased by using a smaller detector aperture, which decreases the sensitivity. The balance between depth resolution, maximum analyzable depth and sensitivity depends on the scientific question and experimental equipment, and a general answer cannot be given.

The proton energy spectrum method was used for depth profiling of deuterium in samples from the main limiter of the tokamak Tore Supra in the framework of the DITS project [23]. One example is shown in Fig. 5. The limiter consists of carbon-fiber composite (CFC) material. At erosion dominated areas of the limiter carbon is eroded, transported by the plasma and redeposited at deposition-dominated areas, where thick codeposited amorphous hydrocarbon layers (a-C:D layers) are formed. The analysis shown in Fig. 5 was done at four different incident energies from 800 to $6000 \mathrm{keV}$. The sample was coated with a very thick layer containing about $20 \% \mathrm{D}$, the rest was almost entirely carbon. The depth profile of $\mathrm{D}$ was derived from the four energy spectra and is shown in Fig 5(bottom), assuming an atomic density of $10^{23}$ atoms $/ \mathrm{cm}^{3}$. The thickness of the deposited layer exceeds the maximum analyzable depth of $38 \mu \mathrm{m}$. Due to the analysis at $4000 \mathrm{keV}$ and $6000 \mathrm{keV}$ without a measurement at an intermediate energy the depth resolution in the depth range from $17-25 \mu \mathrm{m}$ is only $3 \mu \mathrm{m}$ or worse, see Fig. 4 , so that only limited information about the depth profile from this depth range is available. The error bars are due to counting statistics and the quality of the fit. An additional systematic error due to uncertainties of the cross-section, inaccuracies of the beam current measurement, and detector solid angle angle calibration of about $7 \%$ has to be added. For hydrocarbon layers some care is necessary due to ion-beam induced desorption of D. The application of $1 \mu \mathrm{C} 800 \mathrm{keV}{ }^{3} \mathrm{He}$ ions resulted in a decrease of the $\mathrm{D}$ content by about $0.5 \%, 10 \mu \mathrm{C}$ at $2500 \mathrm{keV}$ decreased the content by about $4 \%$, and $10 \mu \mathrm{C}$ at $4000 \mathrm{keV}$ by about $2 \%$ - the detailed numbers depend on deuterium content and thickness of the analyzed layer. As can be seen from the deuterium loss rate, lower energies result in a higher desorption rate. But lower energies also require a smaller collected charge 
due to the higher cross-section, and the desorption takes place in a shallower near-surface layer. Measurements at higher energies following measurements at lower energies therefore detect a partly depleted near-surface layer, and an undisturbed deeper layer. This has to be taken into account when deciding the sequence of measurements at different energies for samples sensitive to ion-induced desorption. Detailed results about the deuterium content of the Tore Supra limiter will be published elsewhere.

\section{Deuterium in tungsten}

While the depth resolution for deuterium in light materials such as carbon is mainly determined by geometrical spread due to finite beam spot size and detector aperture (see section 4), the depth resolution in heavy materials is mainly determined by multiple small-angle scattering. This is shown in Fig. 6 for $2500 \mathrm{keV}$ incident energy. The theoretical tungsten density of $19.29 \mathrm{~g} / \mathrm{cm}^{3}$ was used. Geometrical straggling is the dominant energy-spread component only within the top $250 \mathrm{~nm}$, while in larger depths multiple scattering becomes the main process determining the depth resolution This is due to the angular spread of the incident ${ }^{3} \mathrm{He}$ ions and hence a spread of reaction angles. Multiple scattering of outgoing protons is negligible. Electronic energy-loss straggling in target and stopper foil and the detector resolution have only minor influence. The depth resolution improves for depths exceeding $1.2 \mu \mathrm{m}$ due to the increase of the effective stopping power.

The depth resolution for different incident energies is shown in Fig. 7. For small incident energies the best depth resolution is obtained at the surface, while for energies above about $2000 \mathrm{keV}$ the best depth resolution is obtained deeper inside the sample. As in the case of carbon the highest cross-section and best sensitivity is obtained close to the depth range with optimum depth resolution. A maximum depth of about $11 \mu \mathrm{m}$ can be reached at $6000 \mathrm{keV}$. Because the depth resolution in larger depths is determined by multiple scattering, improvements of the experimental setup (such as a smaller detector aperture or improved detector energy resolution) cannot improve the depth

resolution. For incident energies below about $2000 \mathrm{keV}$ and depths up to about $2 \mu \mathrm{m}$ the proton energy spectrum method offers the better depth resolution. For higher incident energies and larger depths the resonance method is theoretically comparable or superior, but requires measurements at a larger number of incident energies. As in the case of carbon the resolution of the resonance method shown in Fig. 7 is an optimistic limit which may be not fully reached in practical applications. The best depth resolution over the whole analyzable range is obtained by combining both methods and using the proton spectra at incident energies below $2000 \mathrm{keV}$, and the resonance method with an energy step width of $350 \mathrm{keV}$ or less at higher energies. 
An example for depth profiling of $\mathrm{D}$ implanted in plasma-sprayed tungsten is shown in Fig. 8. The sample was exposed at the outer strike point of the tokamak ASDEX Upgrade to a fluence of more than $3 \times 10^{25} \mathrm{D} / \mathrm{m}^{2}$ [4]. The D concentration reaches about 10 at.\% at the surface, most probably due to the formation of a thin hydrocarbon layer. The concentration decreases to about 0.05 at. $\%$ in a depth of about $7 \mu \mathrm{m}$. The simulated spectra are in very good agreement with the experimental ones. Good agreement was also found with depth profiles obtained by SIMS, which will be published elsewhere. The error bars are due to counting statistics and the quality of the fit, and an additional systematic error of about $7 \%$ has to be added. A decrease of the amount of $\mathrm{D}$ by ion-induced desorption was not observed within the count statistics for subsequent analysis of the same beam spot.

\section{Conclusions}

From the possible ion beam analysis methods for quantitative depth profiling of deuterium the $\mathrm{D}\left({ }^{3} \mathrm{He}, \mathrm{p}\right) \alpha$ reaction offers the largest analyzable depth at reasonable depth resolution. This reaction can be exploited for depth profiling by using the resonance-like cross-section maximum at varying incident energies (resonance method), or by measuring the energy spectra of the emitted protons (proton-energy method). With incident ${ }^{3} \mathrm{He}$ energies up to $6000 \mathrm{keV}$ quantitative depth profiles up to about $32 \mu \mathrm{m}$ in carbon and about $11 \mu \mathrm{m}$ in tungsten can be achieved from the energy spectra of the protons. Low deuterium concentrations require sufficiently large detectors to achieve the required sensitivity. This requires a careful selection of detector size and shape for light target materials such as carbon, where the depth resolution is limited by geometrical spread. A reasonable compromise between resolution and sensitivity has to be chosen. For heavy materials like tungsten the resolution is mainly limited by multiple small-angle scattering, which allows the use of larger detectors without additional deterioration of the resolution. A measurement at a single incident energy provides an acceptable depth resolution only in a limited depth range. A reasonable depth resolution throughout the whole analyzed depth can be achieved only by combining measurements at different incident energies. For deuterium in carbon the proton-energy method provides a depth resolution which is better by a factor of more than two compared to the resonance method and needs less different energies. For deuterium in tungsten the proton-energy method provides a better resolution at incident energies below about $2 \mathrm{MeV}$, while at higher energies the depth resolution of the resonance method is superior. A careful selection of incident energies for obtaining an optimum depth resolution throughout the whole analyzed range is required. Due to normal incidence and the high energy of the emitted protons both methods have only a small sensitivity to surface roughness and can 
be used for rough samples. Samples sensitive to ion-induced desorption by the analysis beam (for example hydrocarbons) require a thought-out sequence of measurements at different energies in order to minimize errors due to loss of deuterium. Depth profiling up to very large depths is demonstrated for thick redeposited a-C:D layers on the limiter of Tore Supra and in tungsten coatings from the divertor of ASDEX Upgrade.

\section{Acknowledgements}

The technical assistance by J. Dorner and M. Fußeder is gratefully acknowledged.

\section{References}

[1] J. Roth, V.Kh. Alimov, A.V. Golubeva, R.P. Doerner, E. Tsitrone, Ch. Brosset, V. Rohde, A. Herrmann, and M. Mayer. J. Nucl. Mater. 363-365 (2007) 822.

[2] O.V. Ogorodnikova, J. Roth, and M. Mayer. J. Appl. Phys. 103 (2008) 034902.

[3] T. Loarer, C. Brosset, J. Bucalossi, P. Coad, G. Esser, J. Hogan, J. Likonen, M. Mayer, Ph. Morgan, V. Philipps, V. Rohde, J. Roth, M. Rubel, E. Tsitrone, A. Widdowson, and JET EFDA contributors. Nucl. Fusion 47 (2007) 1112.

[4] M. Mayer, V. Rohde, K. Sugiyama, J.L. Chen, X. Gong, C. Hopf, J. Likonen, S. Lindig, R. Neu, G. Ramos, E. Vainonen-Ahlgren, A. Wiltner, and ASDEX Upgrade Team. J. Nucl. Mater. (2009) . submitted.

[5] J. Likonen, J.P. Coad, E. Vainonen-Ahlgren, T. Renvall, D.E. Hole, M. Rubel, and JET-EFDA Contributors. J. Nucl. Mater. 363-365 (2007) 190.

[6] B.G. Skorodumov, I.O. Yatsevich, V.G. Ulanov, E.V. Zhukovska, and O.A. Zhukovsky. Nucl. Instr. Meth. B 85 (1994) 803.

[7] B.G. Skorodumov, O.I. Buzhinskij, W.P. West, and V.G. Ulanov. J. Nucl. Mater. 233-237 (1996) 1107.

[8] N. Kubota, K. Ochiai, C. Kutsukake, K. Kondo, W.M. Shua, M. Nishi, and T. Nishitani. Fusion Eng. Des. 81 (2006) 227.

[9] J.F. Ziegler, C.P. Wu, P. Williams, C.W. White, B. Terrault, B.M.U. Scherzer, R.L. Schulte, E.J. Schmid, C.W. Magee, E. Ligeon, J. L'Ecuyer, W.A. Lanford, F.J. Kuehne, E.A. Kamykowski, W.O. Hofer, A. Guivarc'h, C.H. Filleux, V.R. Deline, Jr. Evans, C.A., B.L. Cohen, G.J. Clark, W.K. Chu, C. Brossard, R.S. Blewer, R. Behrisch, B.R. Appleton, and D.D. Allred. Nucl. Instr. Meth. 149 (1978) 19. 
[10] P.P. Pronko and J.G. Pronko. Phys. Rev. B9, 7 (1974) 2870.

[11] W. Möller, B.M.U. Scherzer, and R. Behrisch. Nucl. Instr. Meth. 168 (1980) 289.

[12] I.G. Hughes, R. Behrisch, and A.P. Martinelli. J. Nucl. Mater. B64 (1992) 434.

[13] I.G. Hughes, R. Behrisch, and A.P. Martinelli. Nucl. Instr. Meth. B79 (1993) 487.

[14] W.N. Lennard, G.R. Massoumi, P.F.A. Alkemade, I.V. Mitchell, N.S. McIntyre, and R.D. Davidson. Nucl. Instr. Meth. B73 (1993) 203.

[15] V. Kh. Alimov, M. Mayer, and J. Roth. Nucl. Instr. Meth. B 234 (2005) 169.

[16] G. Amsel, F. Pászti, E. Szilágyi, and J. Gyulai. Nucl. Instr. Meth. B, 63 (1992) 421.

[17] D.K. Brice and B.L. Doyle. Nucl. Instr. Meth. B 45 (1990) 265.

[18] E. Szilágyi, F. Pászti, and G. Amsel. Nucl. Instr. Meth. B 100 (1995) 103.

[19] M. Mayer. Nucl. Instr. Meth. B 266 (2008) 1852.

[20] M. Mayer. SIMNRA user's guide. Tech. Rep. IPP 9/113, Max-Planck-Institut für Plasmaphysik, Garching, 1997.

[21] D. Dieumegard, D. Dubreuil, and G. Amsel. Nucl. Instr. Meth. 166 (1979) 431.

[22] J.F. Ziegler. Nucl. Instr. Meth. B 219-220 (2004) 1027.

[23] B. Pégourié, C. Brosset, E. Tsitrone, A. Beauté, S. Brémond, J. Bucalossi, S. Carpentier, Y. Corre, E. Delchambre, C. Desgranges, P. Devynck, D. Douai, G. Dunand, A. Ekedahl, A. Escarguel, E. Gauthier, J. P. Gunn, P. Hertout, S.-H. Hong, F. Kazarian, M. Kočan, F. Linez, Y. Marandet, A. Martinez, M. Mayer, O. Meyer, P. Monier-Garbet, P. Moreau, P. Oddon, J.-Y. Pascal, F. Rimini, J. Roth, F. Saint-Laurent, F. Samaille, S. Vartanian, C. Arnas, E. Aréou, C. Gil, J. Lasalle, L. Manenc, C. Martin, M. Richou, P. Roubin, and R. Sabot. J. Nucl. Mater. (2009) . submitted. 


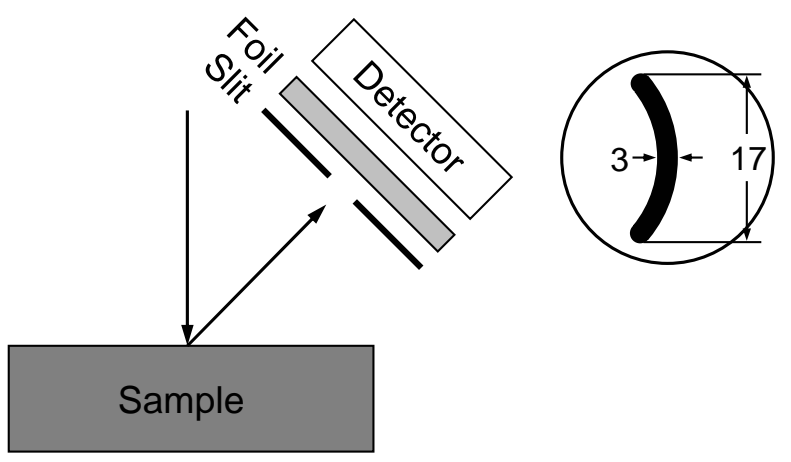

Fig. 1. Schematic representation of the experimental setup. The orientation and size of the detector slit (in $\mathrm{mm}$ ) is indicated.

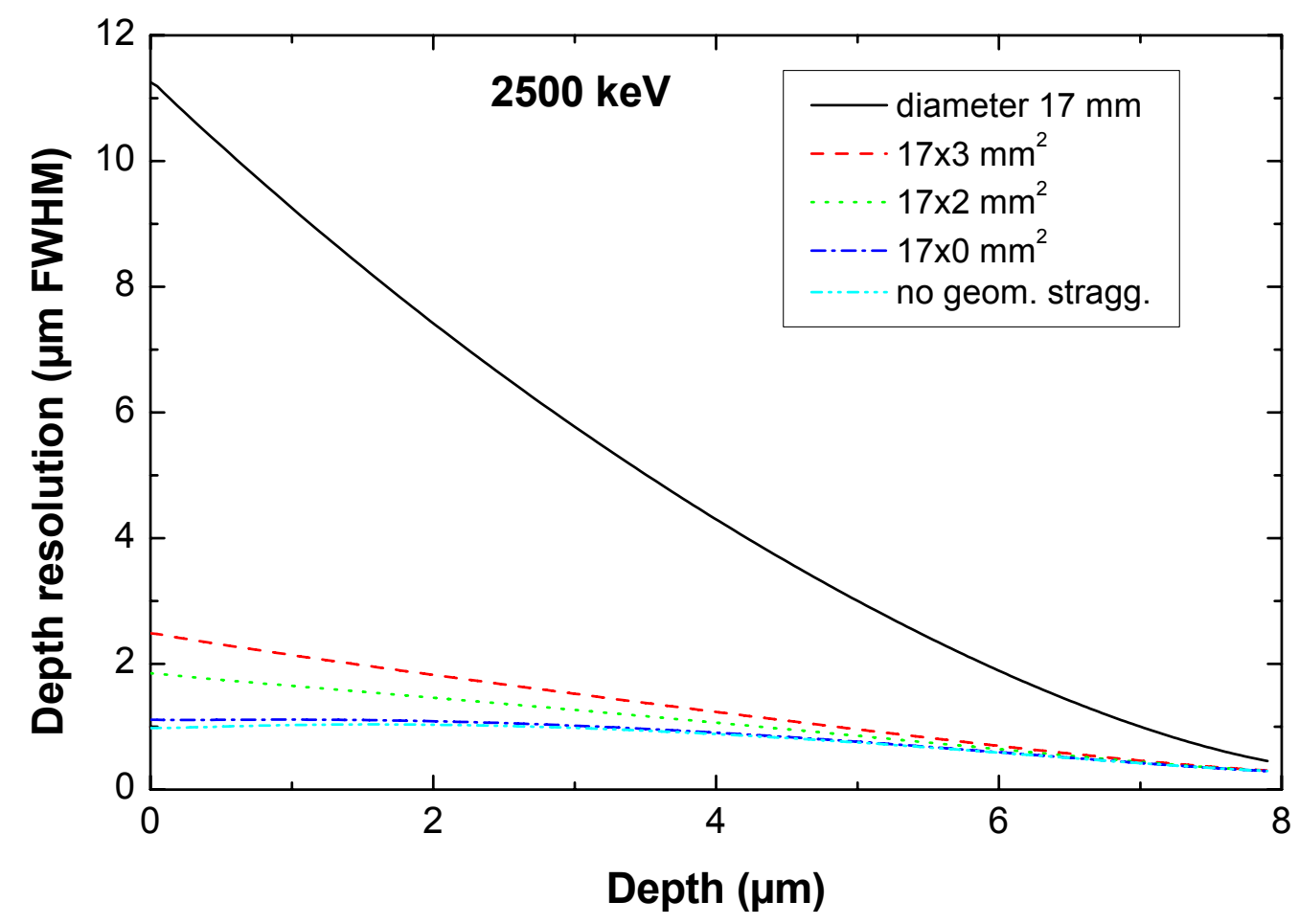

Fig. 2. Depth resolution for deuterium in carbon using the $\mathrm{D}\left({ }^{3} \mathrm{He}, \mathrm{p}\right) \alpha$ reaction at $2500 \mathrm{keV}$ incident energy for different detector apertures: Round detector aperture with diameter $17 \mathrm{~mm}$, parabolic slit in IBM geometry with $17 \mathrm{~mm}$ height and different widths. No geom. stragg.: Without geometrical straggling. Normal incidence, reaction angle $135^{\circ}$, detector-target distance $40 \mathrm{~mm}$, beam spot size $1 \times 1 \mathrm{~mm}^{2}$, detector resolution $22 \mathrm{keV}$, stopper foil $5 \mu \mathrm{m} \mathrm{Ni}$ and $12 \mu \mathrm{m}$ Mylar. 


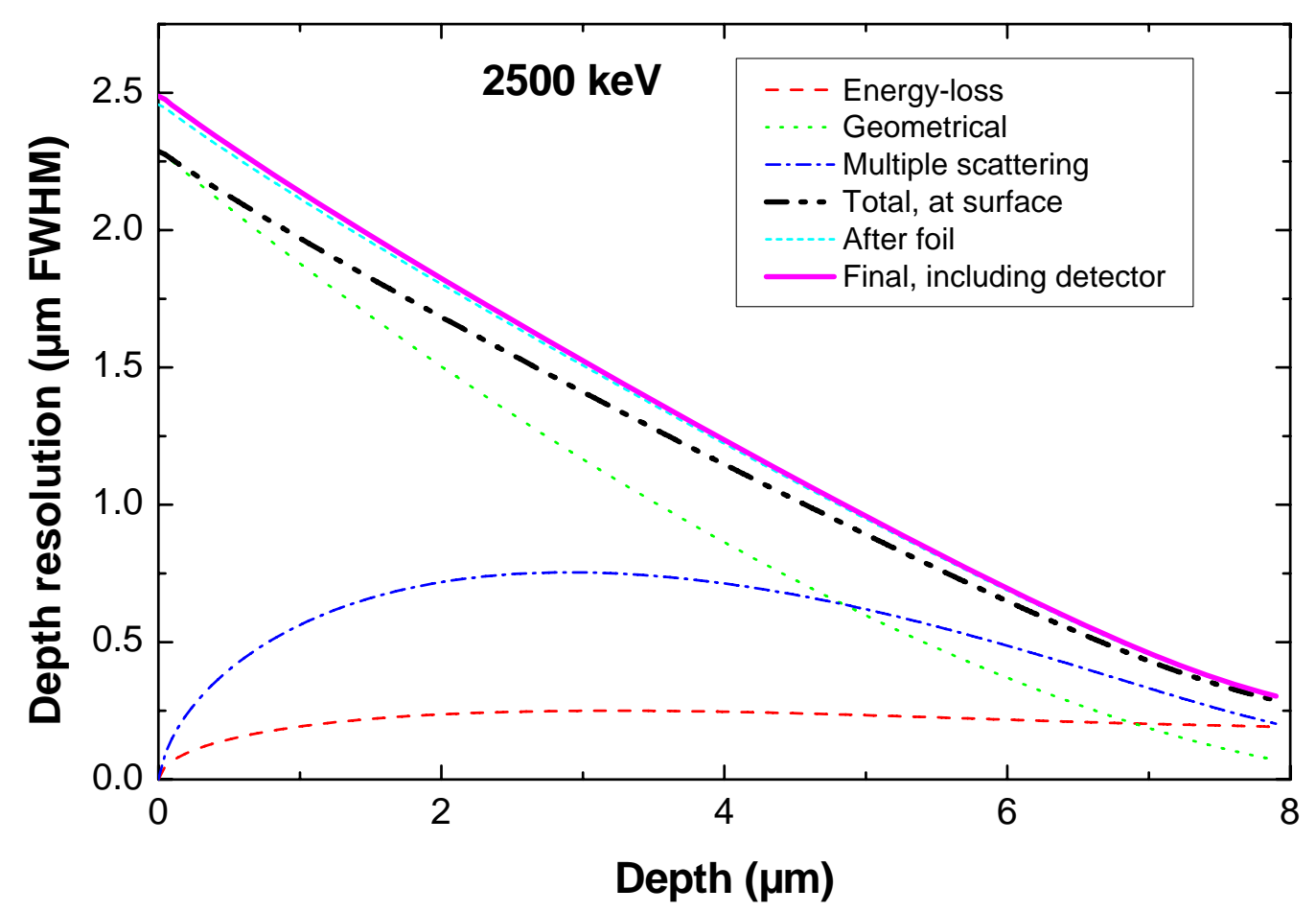

Fig. 3. Contribution of different energy-spread mechanisms to the depth resolution for deuterium in carbon using the $\mathrm{D}\left({ }^{3} \mathrm{He}, \mathrm{p}\right) \alpha$ reaction at $2500 \mathrm{keV}$ incident energy. Dashed line: Energy-loss straggling; Dotted line: Geometrical straggling due to finite detector aperture and beam spot; Dash-dotted line: Multiple small-angle scattering; Thick dash-dot-dot line: Sum of energy-loss straggling, geometrical straggling and multiple scattering at the target surface; Short dashed line: Sum of energy-loss straggling, geometrical straggling and multiple scattering after the stopper foil; Thick solid line: Straggling after the stopper foil plus detector resolution. $2500 \mathrm{keV}$ incident energy, detector aperture $17 \times 3 \mathrm{~mm}^{2}$, other parameters as in Fig. 2 . 


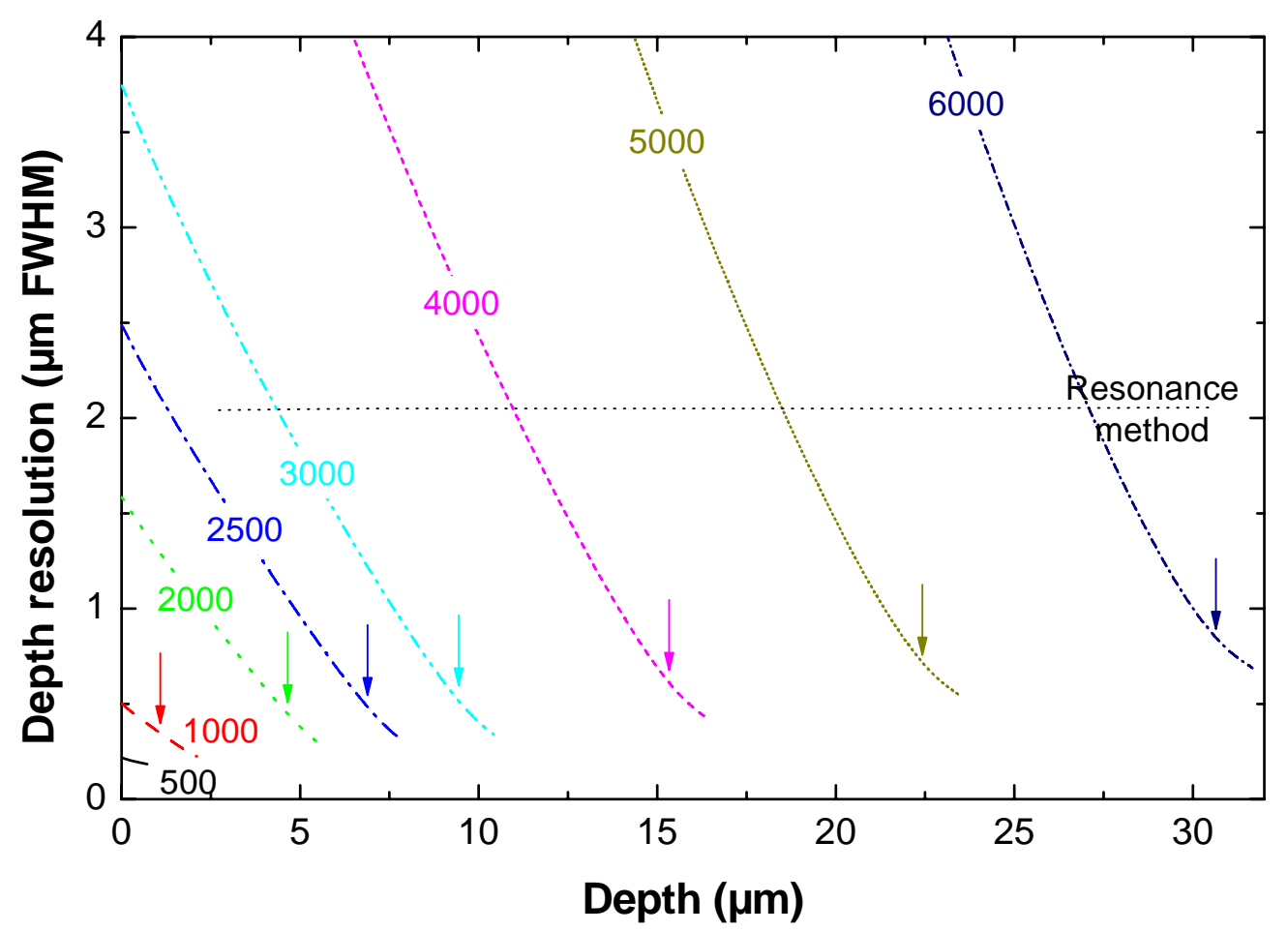

Fig. 4. Depth resolution for deuterium in carbon using the $\mathrm{D}\left({ }^{3} \mathrm{He}, \mathrm{p}\right) \alpha$ reaction at different incident energies. Parameter at curves: Incident energy in keV. Detector aperture $17 \times 3 \mathrm{~mm}^{2}$, other parameters as in Fig. 2. Arrows: Depth at which the mean energy of incident particles has decreased to the maximum of the cross-section. Dotted line: Depth resolution of the resonance method. 

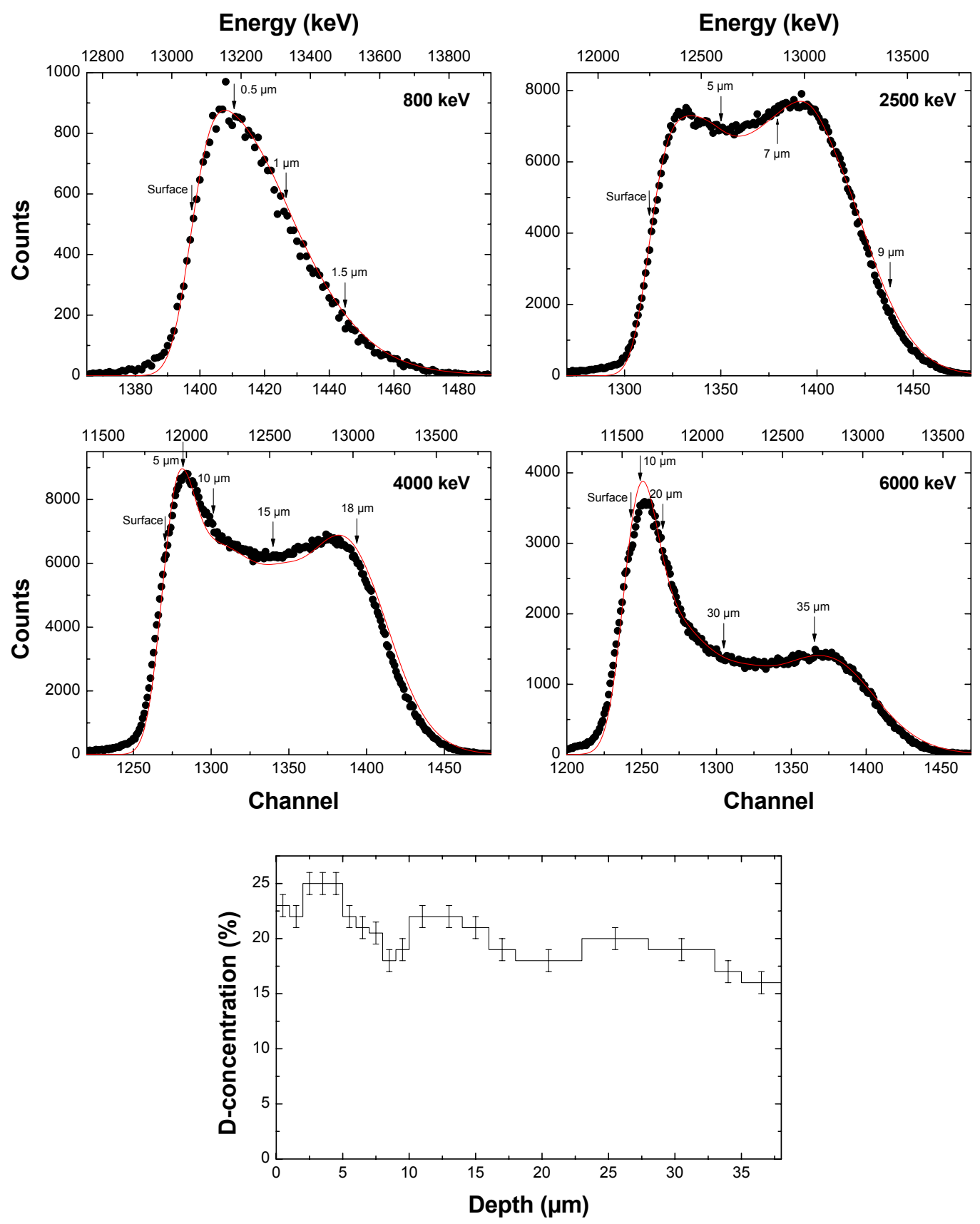

Fig. 5. Proton energy spectra from the $\mathrm{D}\left({ }^{3} \mathrm{He}, \mathrm{p}\right) \alpha$ reaction at 4 different incident energies. The sample was CFC covered with a redeposited a-C:D layer from the tokamak Tore Supra. Dots: Experimental data; Line: SIMNRA simulation; Arrows: Depth of origin of protons with the given energy. Bottom: Deuterium depth profile including error bars using a carbon density of $2 \mathrm{~g} / \mathrm{cm}^{3}$. Applied doses: $800 \mathrm{keV} 1 \mu \mathrm{C}$ ${ }^{3} \mathrm{He}^{+} ; 2500 \mathrm{keV} 10 \mu \mathrm{C}^{3} \mathrm{He}^{+}$; $4000 \mathrm{keV} 10 \mu \mathrm{C}^{3} \mathrm{He}^{+}$; $6000 \mathrm{keV} 5 \mu \mathrm{C}^{3} \mathrm{He}^{2+}$. 


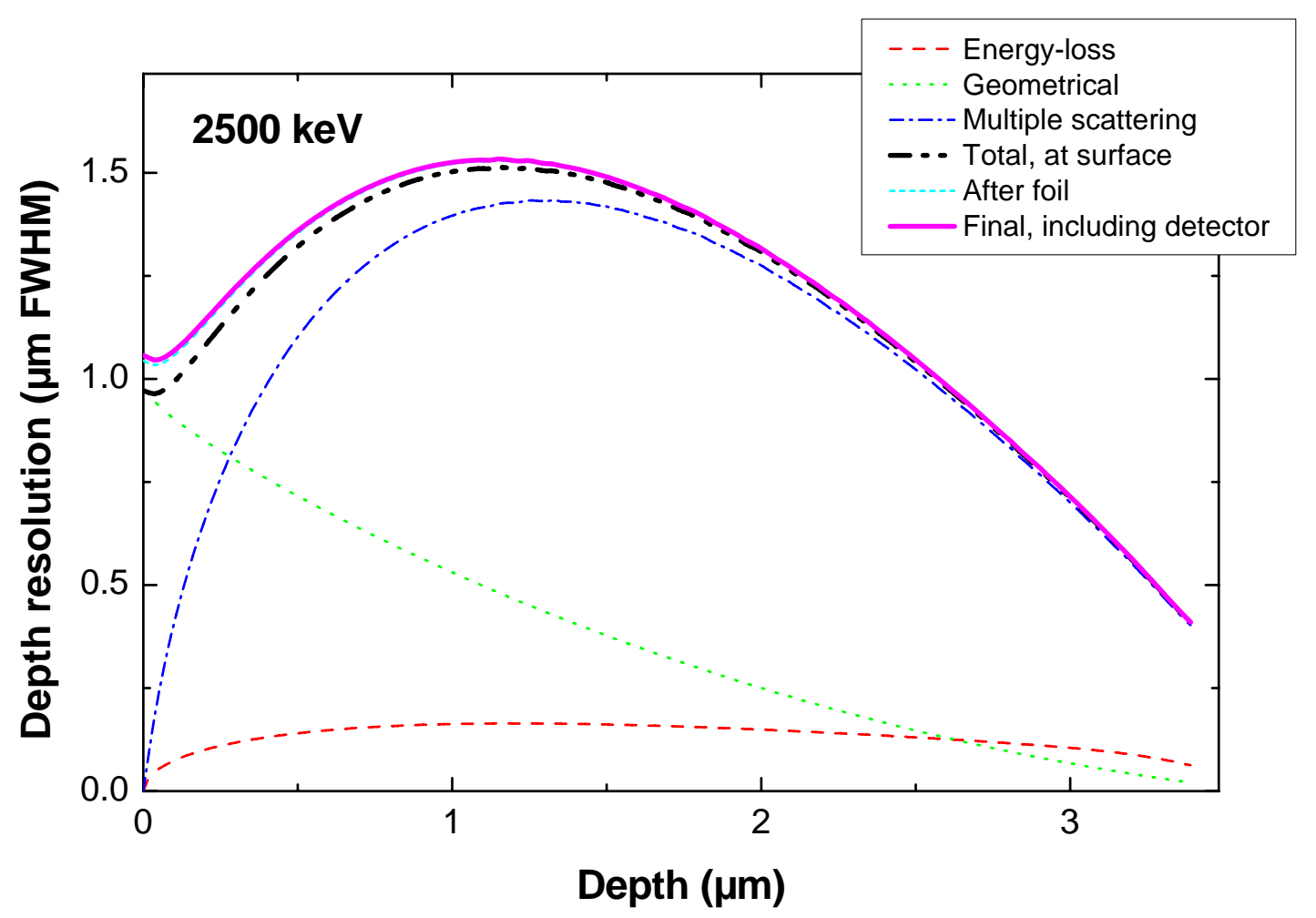

Fig. 6. Contribution of different energy-spread mechanisms to the depth resolution for deuterium in tungsten using the $\mathrm{D}\left({ }^{3} \mathrm{He}, \mathrm{p}\right) \alpha$ reaction at $2500 \mathrm{keV}$ incident energy. Dashed line: Energy-loss straggling; Dotted line: Geometrical straggling due to finite detector aperture and beam spot; Dash-dotted line: Multiple small-angle scattering; Thick dash-dot-dot line: Sum of energy-loss straggling, geometrical straggling and multiple scattering at the target surface; Short dashed line: Sum of energy-loss straggling, geometrical straggling and multiple scattering after the stopper foil; Thick solid line: Straggling after the stopper foil plus detector resolution. $2500 \mathrm{keV}$ incident energy, detector aperture $17 \times 3 \mathrm{~mm}^{2}$, other parameters as in Fig. 2. 


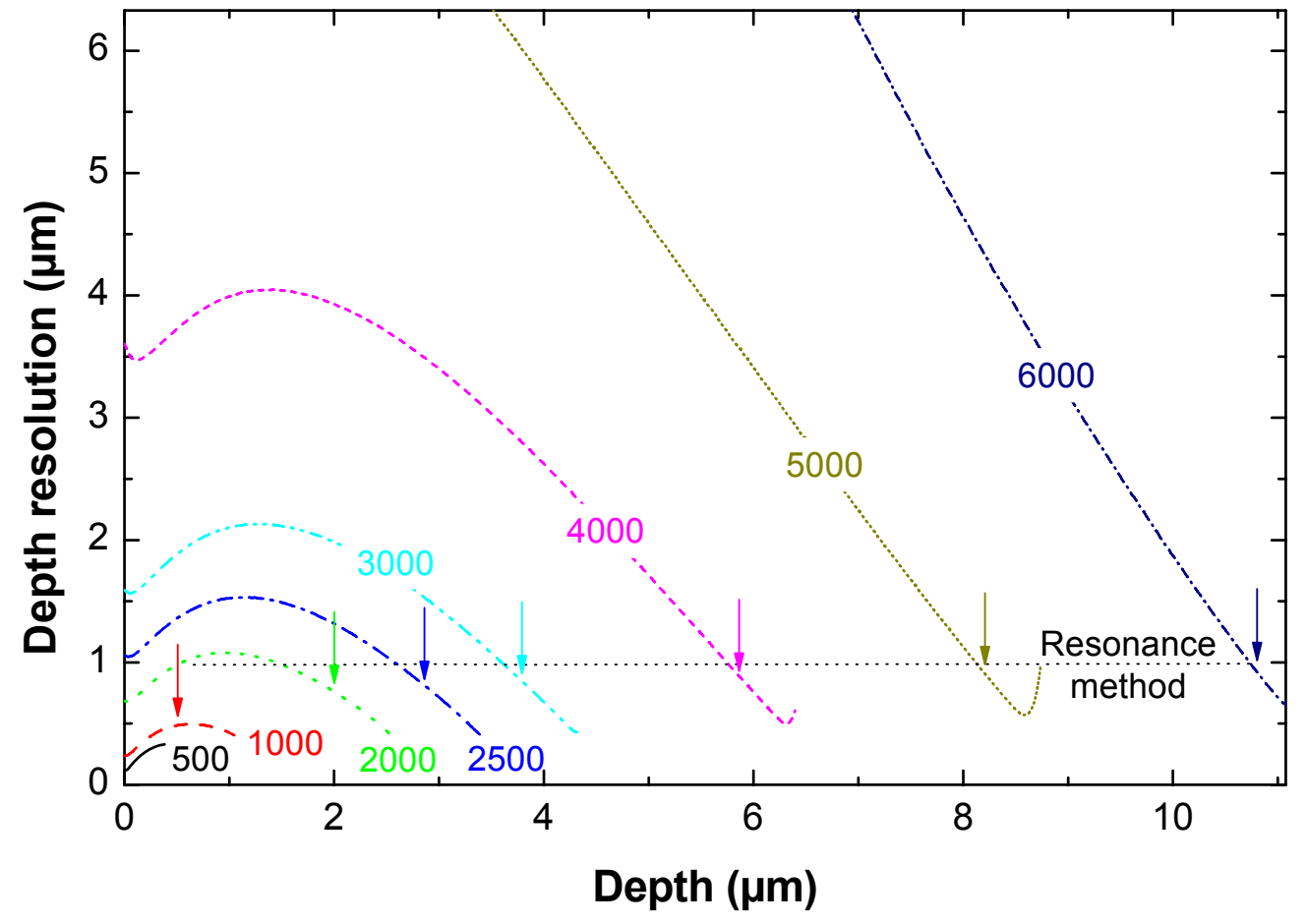

Fig. 7. Depth resolution for deuterium in tungsten using the $\mathrm{D}\left({ }^{3} \mathrm{He}, \mathrm{p}\right) \alpha$ reaction at different incident energies. Parameter at curves: Incident energy in $\mathrm{keV}$. Detector aperture $17 \times 3 \mathrm{~mm}^{2}$, other parameters as in Fig. 2. Arrows: Depth at which the mean energy of incident particles has decreased to the maximum of the cross-section. Dotted line: Depth resolution of the resonance method. 

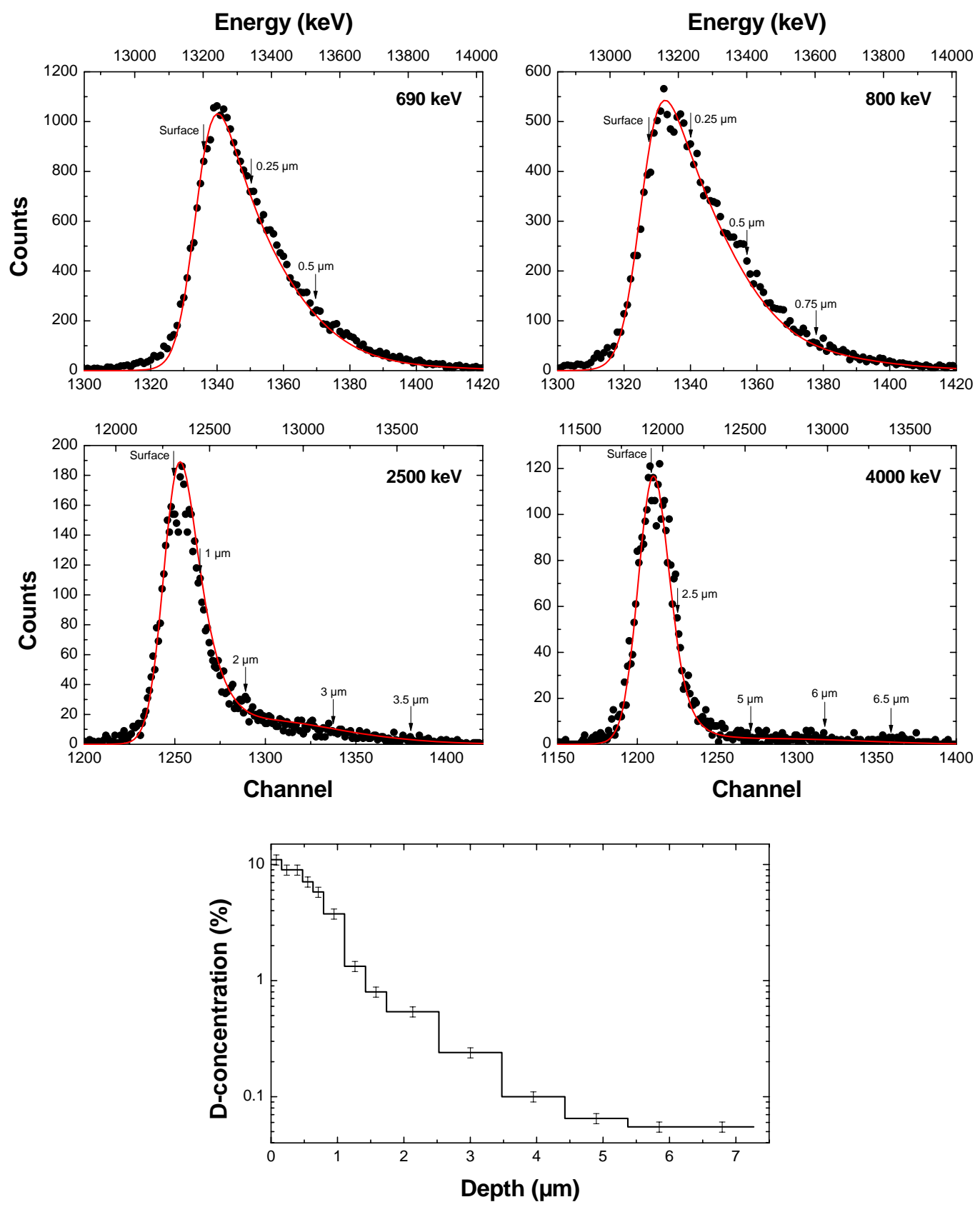

Fig. 8. Proton energy spectra from the $\mathrm{D}\left({ }^{3} \mathrm{He}, \mathrm{p}\right) \alpha$ reaction at 4 different incident energies. The sample was plasma-sprayed tungsten exposed at the outer strike point of the tokamak ASDEX Upgrade. Dots: Experimental data; Line: SIMNRA simulation; Arrows: Depth of origin of protons with the given energy. Bottom: Deuterium depth profile including error bars using a tungsten density of $19.3 \mathrm{~g} / \mathrm{cm}^{3}$. Applied doses of ${ }^{3} \mathrm{He}^{+}: 690 \mathrm{keV} 10 \mu \mathrm{C} ; 800 \mathrm{keV} 5 \mu \mathrm{C} ; 2500 \mathrm{keV} 5 \mu \mathrm{C} ; 4000 \mathrm{keV} 5 \mu \mathrm{C}$. 